\title{
HOLONOMY GROUPS OF COMPLETE FLAT MANIFOLDS
}

\author{
MICHAE SADOWSKI \\ Institute of Mathematics, University of Gdańsk \\ Wita Stwosza 57, 80-952 Gdańsk, Poland \\ E-mail: msa@delta.math.univ.gda.pl
}

\begin{abstract}
We present short direct proofs of two known properties of complete flat manifolds. They say that the diffeomorphism classes of $m$-dimensional complete flat manifolds form a finite set $S_{C F}(m)$ and that each element of $S_{C F}(m)$ is represented by a manifold with finite holonomy group.
\end{abstract}

1. Introduction. The aim of this paper is to give short direct proofs of two fundamental properties of complete flat manifolds (Theorems 1 and 2). A complete flat manifold (cfmanifold) $M$ can be treated as the orbit space $\mathbb{R}^{m} / \Gamma$ of a properly discontinuous and free action of a discrete group $\Gamma$ on a Euclidean space. The holonomy homomorphism $\Phi$ of $M$ carries each element $\gamma$ of the deck group $\Gamma$ onto the linear part of $\gamma$ (cf. [2, p. 51], [11, ch. 3, Lemma 3.4.4]). The group $\Phi(\Gamma)$ is the holonomy group of $M$. We prove the following.

THEOREM 1. Every complete flat manifold is diffeomorphic to a complete flat manifold with finite holonomy group.

THEOREM 2. The set $S_{C F}(m)$ of diffeomorphism classes of complete $m$-dimensional flat manifolds is finite.

Theorem 1 was derived in [10] from related general results. The whole proof presented in [10] is long and difficult. The reader interested in flat manifolds would like to know a shorter one, valid in this particular case. Our proof is more algebraic than that given in [10]. The main step of it consists of showing that an appropriate finite extension of the holonomy group a cf-manifold is a semidirect product of a torsion free abelian group and a finite group (Lemma 2). Theorem 2 has been proved in [10], [8], and [9]. It seems that our elementary proof of it is simpler than the earlier known ones.

2000 Mathematics Subject Classification: Primary 53C25; Secondary 57R22, 20C99.

Key words and phrases: complete flat manifold, holonomy group, flat bundle, diffeomorphism, representation of a Bieberbach group.

The paper is in final form and no version of it will be published elsewhere. 
Theorem 2 cannot be extended to the affine case because the set of affine equivalence classes of noncompact complete flat $m$-manifolds is uncountable ([6, Theorem 1.3] see also [11, ch. 3, Theorem 3.5.1]). This shows that there is a big difference between the theory of closed flat manifolds and the more general theory of complete flat manifolds. The first one can be treated as the theory of Bieberbach groups and the second one uses real representations of these groups (see Section 2 and [6, Theorem 1.2]). For the description of $S_{C F}(m)$ for $m \leq 4$ we refer to [11] and [7].

Throughout this paper the following notation will be used. Given a complete flat manifold $M$, the symbols $\Gamma$ and $\Phi$ mean the same as above. By $X$ we denote the closed totally geodesic submanifold $X$ of $M$ homotopy equivalent to $M$ and by $\Phi_{U}$ the vertical holonomy homomorphism of $M$ (see Section 2 for the definition). The symbols $m$, $n$, and $s$ stand for $\operatorname{dim} M, \operatorname{dim} X$, and $m-n$, respectively. If $G$ is a subgroup of a Lie group $G_{1}$, then $\bar{G}$ is the closure of $G$ in $G_{1}$ and $G_{0}$ is the identity component of $G$. The subgroup of $G$ generated by a subset $S$ of $G$ will be denoted by $\langle S\rangle$.

2. Semidirect products and holonomy groups. The starting point of the proof of Theorem 1 is the description of a complete flat manifold $M$ as the total space of a flat vector bundle over a closed flat manifold. It is known that $M$ contains a closed totally geodesic submanifold $X$ homotopy equivalent to $M$ ([11, Section 3.2]). Let $\Gamma$ be the deck group of $M$ and let $\Phi_{X}$ be the holonomy homomorphism $X$. Write $M$ as $\mathbb{R}^{m} / \Gamma$ and $X$ as $\mathbb{R}^{n} / \Gamma$. The linear isometry $\Phi(\gamma)$ can be written as $\Phi_{X}(\gamma) \times \Phi_{U}(\gamma)$, where $\Phi_{X}(\gamma)$ is an orthogonal transformation of $\mathbb{R}^{n}$ and $\Phi_{U}(\gamma) \in \mathrm{O}(s)$ (see the proof of Theorem 3.3.3 in [11]). The group $\Phi_{X}(\Gamma)$ is always finite and it is isomorphic to the factor group of $\Gamma$ by its maximal abelian subgroup (see e.g. $[11$, ch. $3, \S 3.2, \S 3.4]$ ). In order to describe the holonomy group of $M$ it is necessary to describe its vertical part $\Phi_{U}(\Gamma)$. Let $\Pi: \mathbb{R}^{n} \rightarrow X=\mathbb{R}^{n} / \Gamma$ be the projection and let $\rho: \Gamma \rightarrow \operatorname{GL}(s, \mathbb{R})$ be a representation of $\Gamma$. Consider the diagonal action $\gamma(x, u)=(\gamma x, \rho(\gamma) u)$ of $\Gamma$ on $\mathbb{R}^{n} \times \mathbb{R}^{s}$ and the orbit space $M[\rho]=\left(\mathbb{R}^{n} \times \mathbb{R}^{s}\right) / \Gamma$. The projection of $\mathbb{R}^{n} \times \mathbb{R}^{s}$ onto $\mathbb{R}^{n}$ determines a map $p: M[\rho] \rightarrow X$. The triple $\Pi[\rho]=(M[\rho], X, p)$ is a vector bundle associated to the principal bundle $\Pi$ with typical fiber $\mathbb{R}^{s}$. The arguments given in the proof of Theorem 3.3.3 in [11] show that

LEMMA 1. The manifold $M$ is affinely diffeomorphic to $M\left[\phi_{U}\right]$.

The aim of this section is to prove the following.

LEMMA 2. There is a subgroup $G$ of $\overline{\Phi_{U}(\Gamma)}$ such that:

a) $\Phi_{U}(\Gamma)$ is a finite index subgroup of $G$,

b) $G$ is a semidirect product of a torsion free abelian subgroup $A$ of $\overline{\Phi_{U}(\Gamma)_{0}}$ and a finite group $H$.

Proof. Denote $\Phi_{U}(\Gamma)$ by $G^{*}$ and $\overline{G^{*}}{ }_{0} \cap G^{*}$ by $B$. Let $\Gamma_{m a}$ be the maximal abelian subgroup of $\Gamma$. Since $\Phi_{U}\left(\Gamma_{m a}\right)$ is a finite index subgroup of $\Phi_{U}(\Gamma), \operatorname{dim} \overline{G^{*}}{ }_{0}=\operatorname{dim} \overline{\Phi_{U}\left(\Gamma_{m a}\right)_{0}}$ so that $\overline{G^{*}}{ }_{0}=\overline{\Phi_{U}\left(\Gamma_{m a}\right)_{0}}$ and $\overline{G *}_{0}$ is a torus $T$ covered by a vector space $\widetilde{T}$. For notational convenience the group operations in $T$ will be written additively. If $\rho$ is the order of the torsion subgroup of $B$ and $B_{\rho}=\rho B$, then $B_{\rho}$ is a torsion free normal subgroup of $G^{*}$. Let 
$\mathcal{P}: \widetilde{T} \rightarrow T$ be the projection, $H^{*}=G^{*} / B_{\rho}$, and $r=\left|H^{*}\right|$. Fix a free system of generators $b_{1}, \ldots, b_{l}$ in $B_{\rho}$ and $\widetilde{b}_{j} \in \mathcal{P}^{-1}\left(b_{j}\right)$. Take representatives $h_{1}, \ldots, h_{r}$ of the left cosets of $B_{\rho}$ in $G^{*}$. For $t \in \mathbb{R}, k \in \mathbb{N}$, and $i \in\{1, \ldots, l\}$ consider $b_{i, t}=\mathcal{P}\left(t \widetilde{b}_{i}\right)$, and $b_{i}(k)=b_{i, \frac{1}{k}}$. Let

$$
B(k)=\left\langle b_{1}(k), \ldots, b_{l}(k)\right\rangle \text { and } G(k)=\left\langle B(k), h_{1}, \ldots, h_{r}\right\rangle .
$$

It is easily seen that $B(k)$ is a free abelian group and $k b_{i}(k)=b_{i}$.

Let $g \in G^{*}$ and $I_{g}: x \rightarrow g x g^{-1}$. Since $B_{\rho}$ is a normal subgroup of $G^{*}$, there are $c_{i j} \in \mathbb{Z}$ such that $I_{g}\left(b_{i}\right)=\sum_{j=1}^{l} c_{i j} b_{j}$. In order to prove the equality

$$
I_{g}\left(b_{i, t}\right)=\sum_{j=1}^{l} c_{i j} b_{j, t}
$$

assume that $k$ is relatively prime to $r$. We have

$$
I_{g}\left(b_{i}(k)\right)=\sum_{j=1}^{l} d_{i j} b_{j}(k)+h(i)
$$

for $d_{i j} \in \mathbb{Z}$ and $h(i) \in\left\{h_{1}, \ldots, h_{r}\right\}$. Since

$$
k h(i)=I_{g}\left(k b_{i}(k)\right)-k \sum_{j=1}^{l} d_{i j} b_{j}(k)=\sum_{j=1}^{l}\left(c_{i j}-d_{i j}\right) b_{j} \in B_{\rho}
$$

and $(k, r)=1$ it is clear that $h(i) \in B_{\rho}$. Hence

$$
I_{g}\left(b_{i, \frac{a}{k}}\right)=\sum_{j=1}^{l} c_{i j} b_{j, \frac{a}{k}}
$$

for $a \in \mathbb{Z}$. Now $(*)$ follows from the fact that the set $\left\{\frac{a}{k}: a \in \mathbb{Z},(k, r)=1\right\}$ is dense in $\mathbb{R}$.

Let $H(k)=G(k) / B(k), B(\infty)=\bigcup_{k=1}^{\infty} B(k !), G(\infty)=\bigcup_{k=1}^{\infty} G(k !)$, and $H(\infty)=$ $G(\infty) / B(\infty)$. Clearly $B(\infty) \cong \mathbb{Q}^{l}$. Consider the exact sequence

$$
0 \rightarrow B(\infty) \rightarrow G(\infty) \rightarrow H(\infty) \rightarrow 1
$$

By $\left[1\right.$, ch. 3, Corollary 10.2], $H^{*}(H(\infty), B(\infty))=0$ and this implies that there is a split $\sigma: H(\infty) \rightarrow G(\infty)$.

Every coset of $G(k !)$ modulo $B(k !)$ can be written as $h_{j} B(k !)$ for some $j \in\{1, \ldots, l\}$ so that there is an epimorphism of $H(k !)$ onto $H((k+1)$ !) and consequently $H(k !) \cong H(\infty)$ for $k$ sufficiently big. Fix a positive integer $k_{0}$ such that $\sigma(H) \subset G(k !)$ and $H(k !) \cong H(\infty)$ for $k \geq k_{0}$. Setting $A=B\left(k_{0} !\right), G=G\left(k_{0} !\right)$, and $H=H\left(k_{0} !\right)$, we complete the proof of Lemma 2.

3. Reductions of the holonomy groups of complete flat manifolds. Using the results of Section 2, we complete the proof of Theorem 1.

Proof of Theorem 1. Let $A, G$, and $H$ be as in Lemma 2 and let $T=\bar{A}, \widetilde{T}, \mathcal{P}$, and $\sigma$ be as in the proof of Lemma 2. Take a basis $a_{1}, \ldots, a_{l}$ of the free abelian group $A, t \in[0,1]$, $\widetilde{a}_{j} \in \mathcal{P}^{-1}\left(a_{j}\right)$, and $a_{j, t}=\mathcal{P}\left(t \widetilde{a}_{j}\right) \in T$. The formula $f_{t}\left(a_{j}\right)=a_{j, t}, j=1, \ldots, l$, defines a homomorphism $f_{t}: A \rightarrow T$. 
Let $g \in G$ and $I_{g}: T \ni x \rightarrow g x g^{-1} \in T$. Fix $t \in[0,1]$. As in the proof of Lemma 2, the group operations in $T$ will be written additively. Consider $c_{i j} \in \mathbb{Z}$ such that $I_{g}\left(a_{i}\right)=$ $\sum_{j=1}^{l} c_{i j} a_{j}$. The arguments given in the proof of Lemma 2 show that

$$
I_{g}\left(a_{i, t}\right)=\sum_{j=1}^{l} c_{i j} a_{j, t} .
$$

Hence

$$
I_{g} \circ f_{t}=f_{t} \circ I_{g} .
$$

An element $x$ of $G$ can be uniquely written as $\sigma(h) a$, where $h \in H$ and $a \in A$. Consider the map

$$
F_{t}: G \ni \sigma(h) a \rightarrow \sigma(h) f_{t}(a) \in \bar{G} .
$$

Since $\bar{G}_{0}$ is abelian and $f_{t}\left(\sigma(h) a \sigma(h)^{-1}\right)=\sigma(h) f_{t}(a) \sigma(h)^{-1}$, it is easy to see that $F_{t}$ is a homomorphism.

Let $i: \Phi_{U}(\Gamma) \rightarrow G$ be the inclusion and $\Phi_{U, t}=F_{1-t} \circ i \circ \Phi_{U}$. The bundles $\Pi\left[\Phi_{U, 0}\right]=$ $\Pi\left[\Phi_{U}\right]$ and $\Pi\left[\Phi_{U, 1}\right]$ are isomorphic (compare [3]) so that $M$ is diffeomorphic to the total space $M_{1}$ of $\Pi\left[\Phi_{U, 1}\right]$. Since $\Phi_{U, 1}(\Gamma) \cong H$, the holonomy group of $M_{1}$ is finite. This finishes the proof of Theorem 1 .

ExAMPLE 1. Let $\beta$ be any irrational multiple of $\pi$ and let

$$
f_{\beta}: \mathbb{R}^{3} \ni(x, y, z) \rightarrow(x \cos \beta-y \sin \beta, x \sin \beta+y \cos \beta,-z) \in \mathbb{R}^{3} .
$$

Consider the homomorphism $\rho: \pi_{1}\left(S^{1}\right) \rightarrow \mathrm{O}(3)$ carrying a generator of $\pi_{1}\left(S^{1}\right)$ onto $f_{\beta}$ and $M_{\beta}=M[\rho]$. Then $\Phi_{U}=\rho$ and $\overline{\Phi_{U}(\Gamma)_{0}} \cap \Phi_{U}(\Gamma)=\left\langle f_{\beta}^{2}\right\rangle$, but the arising exact sequence $1 \rightarrow\left\langle f_{\beta}^{2}\right\rangle \rightarrow\left\langle f_{\beta}\right\rangle \rightarrow \mathbb{Z}_{2} \rightarrow 1$ is not split.

4. Diffeomorphism classes of cf-manifolds and flat bundles. Theorem 2 will be derived from the following (cf. [8, Corollary 7.1.5] and [9, Proposition 9]).

LEMMA 3. If $X$ is a closed flat manifold, then the number of isomorphism classes of $s$-dimensional riemannian flat bundles over $X$ is finite.

Proof. The proof of Lemma 3 consists of two steps. In the first we show that there is a finite covering $U_{1}, \ldots, U_{l}$ of $X$ such that any riemannian flat bundle over $X$ admits transition functions constant on each intersection $U_{i} \cap U_{j}$. To achieve this goal, take $\delta \in\left(0, \frac{1}{2} \operatorname{diam}(X)\right)$, smaller than the injectivity radius of $X$, and a $\delta$-net $x_{1}, \ldots, x_{l}$. Let $U_{j}$ be the open geodesic ball of radius $\delta$ with center in $x_{j}$. Clearly $\bigcup_{j=1}^{l} U_{j}=X$. Let $\tau_{c}$ denote the parallel translation along a curve $c$ in $E(\xi)$. By the flatness of $\xi, \tau_{c}$ depends only on the homotopy class of $c$ in the set of paths keeping the endpoints fixed. Fix shortest geodesic segments $c_{i}, i>1$, joining $x_{1}$ to $x_{i}$ and a basis $v_{1}, \ldots, v_{s}$ of $E(\xi)_{x_{1}}$. Consider $v_{k}\left(x_{i}\right)=\tau_{c_{i}}\left(v_{k}\right), x \in U_{i}$, the unique shortest geodesic $c_{x}^{i}$ joining $x_{i}$ to $x$ and

$$
\sigma_{k}^{i}(x)=\tau_{c_{x}^{i}}\left(v_{k}\left(x_{i}\right)\right) .
$$

The local sections $\sigma_{k}^{i}$ determine local trivializations

$$
g_{i}:\left.E(\xi)\right|_{U_{i}} \rightarrow U_{i} \times E(\xi)_{x_{i}}
$$


carrying $\sum_{k=1}^{s} a_{k} \sigma_{k}^{i}(x)$ onto $\left(x, \sum_{k=1}^{s} a_{k} \sigma_{k}^{i}\left(x_{i}\right)\right)$. Let $g_{i j}=g_{j}^{-1} g_{i}$ be the transition functions associated with our local trivializations and let $h_{\xi}$ be the holonomy homomorphism of $\xi$, induced by the flat metric on $\xi$. Clearly $g_{i j}\left(\sigma_{k}^{i}(x)\right)=\sigma_{k}^{j}(x)$ and $h_{\xi}(\gamma)=\tau_{\gamma}$ for $\gamma \in \pi_{1}(X)$.

In order to show that $g_{i j}$ can be written as $h_{\xi}\left(\gamma_{i j}\right)$ note that

$$
\sigma_{k}^{j}(x)=\tau_{c_{x}^{j}}\left(v_{k}\left(x_{j}\right)\right)=\tau_{c_{x}^{j}}\left(\tau_{c_{j}}\left(v_{k}\right)\right) .
$$

The product of paths $\left(c_{x}^{i}\right)^{-1}, c_{i}^{-1}, c_{j}$, and $c_{x}^{j}$ is a loop $d_{x}$. If $\gamma_{i j}$ is the homotopy class of $d_{x}$, then

$$
\sigma_{k}^{j}(x)=\tau_{d_{x}}\left(\sigma_{k}^{i}(x)\right)=\tau_{\gamma_{i j}}\left(\sigma_{k}^{i}(x)\right)=h_{\xi}\left(\gamma_{i j}\right)\left(\sigma_{k}^{i}(x)\right),
$$

as claimed.

Using our covering, it is easy to finish the proof. Let

$$
A=\left\{(i, j) \in\{1, \ldots, l\}^{2}: U_{i} \cap U_{j} \neq \emptyset\right\}
$$

and let $W$ be the set of all maps $\psi: A \rightarrow \mathrm{O}(s)$ such that the function carrying nonempty intersections $U_{i} \cap U_{j}$ onto $\psi(i, j)$ is a cocycle. By the above, each riemannian flat bundle over $X$ admits a cocycle belonging to $W$. The limit of cocycles is a cocycle so that $W$ is a closed subset of the compact set $\operatorname{Map}(A, \mathrm{O}(s))$ and thus $W$ has finitely many connected components.

Proof of Theorem 2. By the third Bieberbach theorem (see [2, ch. 2, Theorem 5.5], [11, ch. 3, Theorem 3.3.2]), the set of diffeomorphism classes of closed flat $k$-manifolds $(k \leq m)$ is finite and thus it suffices to prove that the set of diffeomorphism classes of cf- $m$-manifolds, homotopy equivalent to a fixed closed flat manifold $X$, is finite. This follows immediately from Lemma 3.

REMARK 1. The conclusion of Lemma 3 does not hold for arbitrary manifolds and flat bundles. To see this take a closed orientable surface $X_{g}$ of genus $g$ greater than 1 . By [4, Corollary, p. 215], [5, Appendix C], $X_{g}$ is the base space of a 2-dimensional orientable flat vector bundle $\xi$ whose Euler class $e(\xi)$ belongs to $H^{2}\left(X_{g}, \mathbb{R}\right)-\{0\}$. Given a positive integer $k$ let $\xi^{k}$ be the tensor product of $k$ copies of $\xi$, treated as a complex line bundle. Since $e\left(\xi^{k}\right)=c_{1}\left(\xi^{k}\right)=k e(\xi)$, there are infinitely many isomorphism classes of 2-dimensional flat vector bundles over $X_{g}$.

COROLlARY 1 . There is a constant $C(m)$ such that every $m$-dimensional $c f$-manifold $M$ is diffeomorphic to a cf-manifold $M_{1}$ whose holonomy group has order not greater than $C(m)$.

For an estimate of $C(m)$ we refer to [10, Corollary 6.5]. Wilking's paper contains a direct proof of Corollary 1. It is obvious that Corollary 1 implies Theorems 1 and 2.

\section{References}

[1] K. S. Brown, Cohomology of Groups, Springer, Berlin 1982.

[2] L. Charlap, Bieberbach Groups and Flat Manifolds, Springer, New York, 1986.

[3] W. M. Goldman and M. W. Hirsch, Flat bundles with solvable holonomy, Proc. Amer. Math. Soc. 82 (1981), 491-494. 
[4] J. Milnor, On the existence of a connection with curvature zero, Comm. Math. Helv. 32 (1958), 215-223.

[5] J. Milnor and J. Stasheff, Characteristic Classes, Princeton University Press, Princeton, 1974.

[6] M. Sadowski, Affinely equivalent complete flat manifolds, Cent. Eur. J. Math. 2 (2004), 332-338.

[7] M. Sadowski, Topological and affine classification of complete flat 4-manifolds, to appear.

[8] K. Tapp, The geometry of open manifolds of nonnegative curvature, Ph.D. Thesis, University of Pennsylvania, 1999.

[9] K. Tapp, Finiteness theorems for submersions and souls, Proc. Amer. Math. Soc. 130 (2002), 1809-1817.

[10] B. Wilking, On fundamental groups of manifolds of nonnegative curvature, Differential Geom. Appl. 13 (2000), 129-165.

[11] J. Wolf, Spaces of Constant Curvature, McGraw-Hill, New York, 1967. 